\title{
A comparison of clinical and laboratory manifestations of thrombotic events in patients with COVID-19 and other respiratory viral infections.
}

Chuen Wen Tan ( $\nabla$ tan.chuen.wen@sgh.com.sg )

Singapore General Hospital

Jing Yuan Tan

Singapore General Hospital

Wan Hui Wong

Singapore General Hospital

May Anne Cheong

Singapore General Hospital

Ian Matthias Ng

Singapore General Hospital

Edwin Philip Conceicao

Singapore General Hospital

Guek Hong Jenny Low

Singapore General Hospital

Heng Joo Ng

Singapore General Hospital

Lai Heng Lee

Singapore General Hospital

\section{Research Article}

Keywords: COVID-19, Respiratory viruses, Thrombosis, Clot waveform analysis

Posted Date: September 22nd, 2020

DOI: https://doi.org/10.21203/rs.3.rs-82066/v1

License: (c) (i) This work is licensed under a Creative Commons Attribution 4.0 International License.

Read Full License 


\section{Abstract}

COVID-19 caused by Severe Acute Respiratory Syndrome Coronavirus-2 (SARS-CoV-2) and other respiratory viral (non-CoV-2-RV) infections are associated with thrombotic complications. The differences in prothrombotic potential between SARS-CoV-2 and non-CoV-2-RV have not been well characterised. We compared the thrombotic rates between these two groups of patients directly and further delved into their coagulation profiles. In this single-center, retrospective cohort study, all consecutive COVID-19 and nonCoV-2-RV patients admitted between January $15^{\text {th }}$ and April $10^{\text {th }} 2020$ were included. Coagulation parameters studied were prothrombin time and activated partial thromboplastin time and its associated clot waveform analysis (CWA) parameter, min1, min2 and max2. In the COVID-19 ( $n=181)$ group there were two (1.0 event/1000-hospital-days) thrombotic events while one (1.8 event/1000-hospital-day) was reported in the non-CoV-2-RV $(n=165)$ group. All of these events were myocardial infarction occurring in the intensive care unit. Coagulation parameters did not differ throughout the course of mild COVID-19. However, CWA parameters were significantly higher in severe COVID-19 compared with mild disease, suggesting hypercoagulability $\left(\min 1: 6.48 \% / \mathrm{s}\right.$ vs $5.05 \% / \mathrm{s}, P<0.001 ; \min 2: 0.92 \% / \mathrm{s}^{2}$ vs $0.74 \% / \mathrm{s}^{2}$, $P=0.033)$. In conclusion, the thrombotic rates were low and did not differ between COVID-19 and non-CoV2-RV patients. The hypercoagulability in COVID-19 is a highly dynamic process with the highest risk occurring when patients were most severely ill. Such changes in haemostasis could be detected by CWA. In our population, a more individualized thromboprophylaxis approach, considering clinical and laboratory factors, is preferred over universal pharmacological thromboprophylaxis for all hospitalized COVID-19 patients and such personalized approach warrants further research.

\section{Introduction}

Towards the end of April 2020, investigators were reporting the association of Coronavirus Disease-19 (COVID-19) with increased incidence of thrombotic events. Initially confined to intensive care units (ICU) and critically ill COVID-19 patients, similar findings were subsequently also noticed in non-critically ill patients ${ }^{1,2}$. Emerging autopsy-derived histopathological evidence have confirmed the presence of pulmonary vasculature microthrombosis in deceased patients ${ }^{3,4}$. Thrombotic complication is however not unique to COVID-19. Other non-CoV-2 respiratory viral (non-CoV-2-RV) infections have also been associated with both arterial ${ }^{5,6}$ and venous ${ }^{7}$ thrombosis.

The thrombotic manifestations in COVID-19 patients have led to both the American Society of Hematology (ASH) ${ }^{8}$ and International Society on Thrombosis and Hemostasis (ISTH) ${ }^{9}$ recommending that all hospitalized patients including the non-critically ill, receive venous thromboembolism (VTE) thromboprophylaxis with low molecular weight heparin (LMWH) or fondaparinux. It is uncertain if such recommendations should be universally adopted as there is significant heterogeneity in reported thrombotic rates as most studies were carried out on critically ill COVID-19 patients ${ }^{10}$. Additionally, 
baseline VTE risk is known to differ between geographical regions, race and ethnicity ${ }^{11,12}$. As the absolute risk of bleeding increases by around $0.5 \%$ with thromboprophylaxis ${ }^{13}$, stratifying COVID-19 patients with high risk of VTE for thromboprophylaxis is crucial to optimize the risk benefit ratio. Measurable laboratory parameters such as elevated D-dimer and fibrinogen levels have been reported to be associated with thrombosis in COVID-19. Less accessible global hemostatic assays such as thromboelastogram and clot waveform analysis (CWA) have also demonstrated hypercoagulability, albeit in small groups of critically ill COVID-19 patients. ${ }^{14,15}$

In this study, we aimed to determine the thrombotic rates in consecutive COVID-19 patients compared to patients with non-CoV-2-RV infections and to evaluate the dynamic haemostatic changes in both groups of patients using global coagulation assays.

\section{Methods}

This single-center study was conducted at Singapore General Hospital, a 1700-bedded tertiary academic center.

For our first objective, we performed a retrospective cohort study to determine thrombotic event rates in all consecutive COVID-19 patients compared to patients diagnosed with non-CoV-2-RV from January $17^{\text {th }}$ to April $10^{\text {th }} 2020$. During this period, all patients who fulfilled our hospital's COVID-19 suspect criteria were admitted regardless of disease severity and tested for both COVID-19 and other respiratory viral pathogens ${ }^{16}$. COVID-19 testing was performed with reverse transcriptase polymerase chain reaction (rtPCR) on RNA extracted from either oropharyngeal and/or nasopharyngeal swabs specimens. Testing for other respiratory viral pathogens was performed using a 16-target respiratory viral PCR assay (Respiratory syncytial virus A/B, Influenza A/B, Parainfluenza viruses 1-4, Metapneumovirus, Rhinovirus A/B/C, Human Coronavirus OC43/229E/NL63, Adenovirus, Human Enterovirus, Human Bocavirus 1-4) on oropharyngeal specimens. Exemption of consent was granted by our institutional review board as this study only involved collection and analysis of routine clinical data (CIRB no. 2020/2535). Study data was obtained from our Hospital's Department of Infection Prevention and Epidemiology database. This database consists of de-identified data collected from all confirmed and suspect COVD-19 cases admitted to our hospital during this pandemic period. Data on demographics, co-morbidities, laboratory coagulation profile parameters, and anti-platelet/anticoagulation medications were obtained. Information on modality of thromboprophylaxis (mechanical vs routine chemical) initiated, thrombotic events during hospitalization, the need for high dependency unit (HDU) and intensive care unit care (ICU), length of stay in ICU and length of hospitalization was also obtained. Need for oxygen supplementation during hospital admission was also collected and was used as a surrogate measure for disease severity in our analysis. We included both arterial and venous thrombotic events. Venous thrombotic events were defined as any venous thromboembolism including deep vein thrombosis, pulmonary embolism and thrombosis of other 
sites, which were objectively confirmed on radiological imaging after initial clinical suspicion by attending physicians. Arterial events were defined as either myocardial infarction or stroke.

For our second objective, laboratory coagulation tests data were pooled from two sources: a) both COVID19 and non-CoV-2-RV patients as studied above and b) COVID-19 patients from our hospital's infectious disease department novel pathogen study database (CIRB no. 2018/3045) censored at $1^{\text {st }}$ June 2020. This database is part of a prospective study to characterize emerging infectious diseases and was approved by our institutional review board (CIRB no. 2018/3045). We collected the following coagulation data: prothrombin time (PT), activated partial thromboplastin time (aPTT) and its associated clot waveform analysis (CWA), fibrinogen and D-dimer.

Coagulation tests taken when patients were on anticoagulants or on citrate-based haemodialysis and patients with active malignancy or pregnancy were excluded from any inter-individual comparison and analysis. APTT-based CWA were generated during the analysis of the standard aPTT assay triggered with Dade Actin FSL reagent (Siemens Healthcare, Marburg, Germany) and retrieved from CS2100i automated coagulation analysers (Sysmex Corporation, Kobe, Japan). The three CWA parameters of interest were min1, min2 and max2 denoting the maximum velocity, maximum acceleration and maximum deceleration of the clot formation process, respectively.

\section{$\underline{\text { Statistical analysis }}$}

Categorical variables were presented as frequencies (percentages) and continuous variable was presented as median (interquartile range) due to its skewed distribution. Univariate analysis was performed using chi square test for categorical variables and Mann Whitney $\mathrm{U}$ test for continuous variables. Correlation analysis was performed using Spearman's rank correlation coefficient $\left(R_{S}\right)$. All tests were two sided, with $\mathrm{p}$-values of $P<0.05$ considered statistically significant. Data analysis was performed using SPSS 25.0 (IBM SPSS statistics, IBM Corporation, USA) software.

\section{Results}

During the study period, a total of 181 patients testing positive for SARS-CoV-2 and 165 patients positive for non-CoV-2-RV via rt-PCR testing. The respiratory viruses were rhinovirus $(n=65)$, influenza $A$ and $B$ $(n=46)$, adenovirus $(n=13)$, human coronavirus 229E/NL63/OC43 $(n=15)$, human enterovirus $(n=3)$, metapneumovirus $(n=6)$, parainfluenza virus 1 to $4(n=11)$ and respiratory syncytial virus $(n=6)$. Among the 186 COVID-19 patients, four patients were co-infected with other respiratory viruses and were excluded from analysis. Age and comorbidities were not significantly different between the two groups 
(Table 1). Compared to the non-CoV-2-RV arm, there were significantly higher proportion of male patients and patients of Indian ethnicity in the COVID-19 arm. Majority (94\%) of our COVID-19 patients were managed in the general isolation wards and had relatively mild infection as reflected by low proportions requiring any form of oxygen supplementation (11\%). COVID-19 patients had longer length of hospitalization and more required ICU support. Mortality rate was low in both groups. The two deaths in our COVID-19 cohort were due to severe COVID-19 pneumonia with multi-organ failure.

\section{Thrombotic outcomes}

In both groups, all general ward patients were not given routine thromboprophylaxis. There was no standardized hospital practice with regards to pneumatic calf pumps for general ward patients and thus such data was unavailable. Patients in HDU were only on mechanical thromboprophylaixs (TED stockings). In contrast, patients in ICU were all started on prophylactic subcutaneous enoxaparin 40mg once a day (or $20 \mathrm{mg}$ once a day for patients with renal failure) unless contraindicated, together with pneumatic calf pumps. Amongst our COVID-19 ICU patients, two (22.2\%) out of nine patients were not on prophylactic thromboprophylaxis because of thrombocytopenia and anemia respectively. There were two arterial thrombotic events in the COVID-19 group, both of which occurred while patients were in ICU. This corresponded to a $22.2 \%$ incidence amongst COVID-19 ICU patients and $1.1 \%$ incidence amongst all COVID-19 patients. On the other hand, there was only one arterial thrombotic event ( $0.6 \%$ incidence) in the non-CoV-2-RV group and this occurred while the patient was in the general ward. The thrombotic rates in COVID-19 and non-CoV-2-RV patients adjusted for the duration of hospitalization translated to 1 per 1000 patient-days and 1.8 per 1000 patient-days respectively. There were no VTE events in both groups. Only one patient had disseminated intravascular coagulopathy (DIC) by ISTH criteria ${ }^{17}$ and sepsis induced coagulopathy (SIC) by criteria proposed by Iba et al ${ }^{18}$. This occurred on day 26 of symptoms and day 21 of ICU stay at which time there was an ongoing component of ventilator associated pneumonia and there was no occurrence of thrombotic event during the study period.

Both cases of arterial thrombosis in the COVID-19 group were non-ST elevation myocardial infarction (NSTEMI). Both occurred early in the course of disease, at day 9 and day 12 of symptom onset respectively. The arterial thrombosis in our non-CoV-2-RV cohort was also a NSTEMI, developing on day 4 of admission. All of them were on prophylactic enoxaparin at the time of thrombosis. There was only one major bleeding event due to intracranial haemorrhage which occurred in the ICU in our entire cohort.

\section{Hemostatic assays}

The coagulation profiles and CWA parameters were analysed. 42 COVID-19 and 5 non-CoV-2-RV patients had only a single data point. 14 COVID-19 patients had data available on two or more time points while only two of the non-CoV2-RV patients had serial coagulation profile data, both of whom were critically ill. 
No biphasic waveform was noted in all the clot waveform curves analysed. The key findings are summarized here:

a) Coagulation functions remained fairly stable in mild COVID-19 and not significantly different from baseline (convalescent state) within the same individuals (Table 2)

In COVID-19 patients who remained clinically well without a need for supplemental oxygen, intraindividual paired comparison of PT, aPTT and CWA data across various time points were not significantly different throughout the course of the disease. Paired comparison between the initial and convalescent coagulation tests also did not show remarkable alteration.

In addition, inter-individual comparison between the presenting coagulation assays taken from patients with mild COVID-19 and coagulation assays taken at convalescent phase also supported this observation - although aPTT was shorter in convalescent phase, CWA profiles and PT were not significantly different between the two groups (Supplementary Table 1).

b) CWA parameters increased with clinical deterioration of COVID-19 (Table 3 and Figure 1)

Comparing the PT, aPTT and CWA data between COVID-19 patients with clinically mild disease and COVID-19 patients who required supplementary oxygen support, a surrogate of more severe disease, aPTT was significantly more prolonged and CWA parameters, especially min1, were significantly elevated in patients with severe disease. PT and D-dimer results did not differ significantly between the two groups.

Serial PT, aPTT and CWA data depicting the various stages, from initial presentation through clinical deterioration to recovery and convalescence were available in one patient and the dynamic changes are shown in Figure 1. Initial clinical deterioration was associated with prolongation of aPTT but aPTT normalised during the subsequent course of ICU stay and remained so at convalescent phase of COVID19. However, min 1 showed marked elevation upon ICU admission and continued to rise throughout the ICU stay despite normalisation of aPTT. Min1 only started to decrease at convalescence and eventual normalised on the $46^{\text {th }}$ day from symptoms onset. Min2 and max2 demonstrated similar pattern of changes to $\min 1$ although the timing of their increment to the levels beyond the respective normal ranges was delayed compared to min1. This patient's PT levels remained normal throughout.

c) Critically ill COVID-19 patients CWA showed a trend of having higher CWA parameters as compared to critically ill patients with non-CoV-2-RV. (Table 4)

Patients with mild COVID-19 and those with non-CoV-2-RV had similar PT, aPTT and CWA profiles. In contrast, for patients who required ICU admission, critically ill COVID-19 patients CWA showed a trend of having higher CWA parameters compared to similar patients with non-CoV-2-RV, although their PT, aPTT and platelet counts were not different. 
Paired analysis data, available in a limited number of subjects, comparing intra-individual CWA fluctuations before and after clinical deterioration demonstrated distinct pattern of change between COVID-19 and non-CoV-2-RV infections. In two non-CoV-2-RV patients, their median PT (10.65s vs 11.10s) and aPTT (37.30s vs $41.45 \mathrm{~s}$ ) became longer whereas their CWA profiles decreased (min $1: 5.80 \% / \mathrm{s}$ vs $4.57 \% / \mathrm{s} ; \min 2: 0.79 \% / s^{2}$ vs $0.48 \% / s^{2} ; \max 2: 0.58 \% / s^{2}$ vs $0.39 \% / s^{2}$ ) with clinical deterioration. The four pairs of data in COVID-19 patients also showed that their PT (10.35s vs 11.20s) and aPTT (40.20s vs 41.40s) were prolonged with clinical deterioration. However, in contrast to non-CoV-2-RV patients, their CWA parameters became elevated instead $\left(\min 1: 5.82 \% / \mathrm{s}\right.$ vs $6.16 \% / \mathrm{s} ; \min 2: 0.74 \% / \mathrm{s}^{2}$ vs $0.81 \% / \mathrm{s}^{2}$; $\max 2: 0.54 \% / s^{2}$ vs $0.62 \% / s^{2}$ ) with increasing severity of infection.

\section{d) CWA parameters correlated with D-dimer and fibrinogen}

Based on ISTH recommendations, D-dimer of six folds the upper limit of normal in COVID 19 patients was a predictor of thrombotic events and thus may be used as a surrogate marker for consideration of higher dose of thromboprophylaxis ${ }^{9}$. Eight COVID-19 patients had D-dimer levels of greater than a cut-off of $3.00 \mathrm{mg} / \mathrm{L} \mathrm{FEU} \mathrm{(approximately} \mathrm{six} \mathrm{folds} \mathrm{of} \mathrm{the} \mathrm{upper} \mathrm{normal} \mathrm{limit).} \mathrm{Of} \mathrm{these,} \mathrm{six} \mathrm{patients} \mathrm{had} \mathrm{severe}$ disease requiring oxygen support, one had mild disease and one was in convalescent phase of COVID-19. Of the two COVID-19 cases with thrombotic complications, only one had a high D-dimer of $4.33 \mathrm{mg} / \mathrm{L} \mathrm{FEU}$ while another had a level of $1.22 \mathrm{mg} / \mathrm{L} \mathrm{FEU}$ at the time when the thrombotic events occurred.

Amongst COVID-19 patients, 35 sets of data were available for correlation evaluation with D-dimer (Supplementary Table 2). While PT and aPTT did not correlate with D-dimer $\left(R_{S}=0.153, P=0.143\right.$ and $R_{S}$ $=0.183, P=0.292$, respectively), CWA parameters correlated significantly with $\mathrm{D}$-dimer $\left(\min 1: \mathrm{R}_{\mathrm{S}}=0.476\right.$, $\left.P=0.004 ; \min 2: \mathrm{R}_{\mathrm{S}}=0.355, P=0.037 ; \max 2: \mathrm{R}_{\mathrm{S}}=0.282, P=0.101\right) .21$ sets of data were analysed for correlation with fibrinogen (Supplementary Table 2). With regards to fibrinogen, PT and aPTT showed no correlations (PT: $\mathrm{R}_{\mathrm{S}}=-0.045, P=0.847$; aPTT: $\left.\mathrm{R}_{\mathrm{S}}=0.269, P=0.239\right)$ but all CWA parameters demonstrated significant positive correlations $\left(\min 1: \mathrm{R}_{\mathrm{S}}=0.812, P=<0.001 ; \min 2: \mathrm{R}_{\mathrm{S}}=0.767, P<0.001\right.$; $\left.\max 2: \mathrm{R}_{\mathrm{S}}=0.695, P=<0.001\right)$.

\section{Discussion}

The latest CHEST guidelines on VTE and its management in COVID -19 patients have provided more mature data on VTE rates ${ }^{10}$. Overall, venous thrombotic events are estimated to be around $8 \%$ in all COVID-19 patients and as high as $69 \%$ in critically ill patients. Other respiratory viral infections such as influenza are also known to be associated with thrombotic events ${ }^{7,19}$ with a $5.9 \%$ prevalence reported during the 2009 influenza pandemic ${ }^{20}$. Presently, indirect evidence and observations seem to suggest a higher thrombotic rate amongst COVID-19 patients compared to patients infected with other respiratory viruses ${ }^{21}$. However, direct comparison of thrombotic events between concurrent cohorts of COVID-19 and non-CoV-2-RV patients is lacking. 
In contrast to other recent reports $2,22,23$, our findings did not show increased VTE events in either groups. This could be accounted by a number of factors peculiar to our study population. At the time of study, there was a large outbreak involving migrant workers residing in dormitories and all COVID-19 cases regardless of the severity were required to be hospitalized according to national policy and hence the majority of our patients were younger with less comorbidities and had a lower rate of ICU stay. Older age, presence of cardiovascular risk factors and ICU stays are all independent risk factors for VTE. In ICU patients with COVID-19, other studies have reported up to $69 \%$ VTE incidence ${ }^{24,25}$. The stark difference in our population may also suggests the influence of other factors such as racial predilection. Whilst local data show that the incidences of VTE are comparable amongst the major ethnic groups of Chinese, Indian and Malay ${ }^{26}$, Asians in general are purported to have lower risk of VTE compared to Caucasians ${ }^{27-}$ ${ }^{29}$ although the actual reasons for this difference are still unclear. In our center, VTE screening was not done as a routine for asymptomatic cases. Confirmatory imaging for VTE would only be performed for symptomatic or clinically suspicious cases. As such our VTE detection rates may be lower compared to centers that practice routine screening of their patients. Albeit the variations in threshold or protocols for detecting VTE in the various studies, it is compelling that our thrombotic rates were clearly much lower compared to other centers.

COVID-19 and other acute respiratory viruses have both been reported to be associated with higher risk of arterial thrombotic events as well. Acute coronary syndrome is reported in no greater than $3 \%$ of acute influenza or other viral infections ${ }^{5,30}$ whilst in COVID-19, the rates vary from $3.4 \%$ to $11.2 \% 21,31$. We, however, reported low arterial event rates in our patient cohorts and the potential reasons accounting for these differences are likely to be similar to those discussed above for VTE. In our cohort, the arterial events occurred only in ICU when the patients were sickest while no ischemic events were reported in our patients from the general ward. Nonetheless, early recognition of cardiac complications in both COVID19 and other viral infections cannot be over-emphasized to reduce morbidity and mortality.

We also evaluated the coagulation profiles of our COVID-19 patients across the different spectrum of disease severity. Commensurate with the absence of thrombotic events observed, none of our mild COVID-19 cases demonstrated evidence of DIC or SIC and their CWA parameters were not elevated. Marked elevation of D-dimer, defined as six-times upper limit of normal, was suggested by ISTH as a possible consideration for intensification of prophylactic pharmacological anticoagulation in COVID-19 9 . However, this cut-off was neither sensitive nor specific for thrombotic events in our cohort as none of our patients with markedly raised D-dimers developed venous thromboembolic event and only one of them suffered an arterial event. 
To further evaluate the thrombotic potential in COVID-19, we analysed available CWA parameters as a global haemostatic assessment. It has been reported that CWA parameters differ in various types of infections $^{32}$ and increased CWA is associated with hypercoagulability ${ }^{33}$. Our findings suggest that there were no significant changes in the overall haemostatic functions in mild COVID-19 but severe COVID-19 was associated with a prothrombotic state and this hypercoagulability gradually normalizes during the convalescent phase. Although CWA parameters correlated with D-dimer and fibrinogen, the strengths of association were weak and moderate respectively and this suggests that the haemostatic functions measured by these assays do not replicate each other. Hence, composite data from these multiple assays might provide more insight into the overall haemostatic dysfunctions of COVID-19 patients. These assays may therefore complement each other to better risk stratify the thrombotic risk of COVID-19 patients which may guide thromboprophylaxis management. Nonetheless, the coagulation assays in this study only evaluated the plasmatic components of haemostasis. Endothelial functions and cellular components, including platelets and leucocytes, and fibrinolytic functions are not assessed but may contribute significantly to coagulation changes in COVID-1934,35.

ISTH has recommended for a universal strategy of routine thromboprophylaxis for all hospitalized COVID-19 patients with standard dose LMWH or unfractionated heparin after assessment of bleeding risk. While existing literature do suggest a significantly high rate of thrombotic events in COVID-19 patients, and understandably warrants the recommendation for thromboprophylaxis, our cohort study has not proven the case in our population. As routine thromboprophylaxis is not without risks, the adoption of this strategy for all hospitalised COVID-19 patients may not be universally suitable. Adjustments to these recommendations should be made taking into account the local population profile and prevalence of thrombotic events. Irrefutably, analysis of hemostatic parameters showed a hypercoagulable state in patients with more severe COVID-19 and thus supporting the use of thromboprophylaxis in critically ill COVID-19 patients.

Although our study has several limitations due to its retrospective nature and the number of thrombotic events in both groups were small thus limiting statistical comparison, we believe our comparison data are sufficiently robust as we included all consecutive patients with the same admitting criteria within the same study period and the demographics between both groups were comparable.

\section{Conclusion}


The thrombotic rates in both hospitalised COVID-19 and non-CoV-2-RV patients were low and did not seem to differ significantly between the two groups. A more individualized thrombotic risk assessment and management approach, possibly involving the combination of various clinical factors and coagulation markers, is an area of unmet needs and warrants further research works.

\section{Declarations}

\section{Acknowledgments}

We acknowledge the extraordinary work of Singapore General Hospital clinical teams who work tiredlessly in our emergency department, isolation wards and acute respiratory infection wards. Our study could not have been done without their meticulous efforts in history taking and clinical documentation. We would also like to acknowledge Singapore General Hospital Department of Infection Prevention and Epidemiology in providing the clinical data for the manuscript.

\section{Authorship Contributions}

CWT and JYT had full access to all the data in the study and takes responsibility for the integrity of the data and accuracy of data analysis. CWT and JYT contributed to statistical analysis, data interpretation and drafting of manuscript. CWT, JYT, LHL, HJN, JGL conceived the study. CWT, JYT, IMN, EPC contributed to acquisition data. LHL and HJN contributed to critical revision of manuscript. All authors agreed to be accountable for all aspects of the work in ensuring that questions related to the accuracy or integrity of any part of the work are appropriately investigated and resolved.

\section{Conflict of Interest Disclosures}

We declare no competing interests.

\section{Funding}

This research was funded by the SingHealth Duke-NUS Academic Medicine COVID-19 Research Grant.

\section{Data availability}

The data used and analyzed in this study are available from the corresponding author on reasonable request. 


\section{References}

1 Cui, S., Chen, S., Li, X., Liu, S. \& Wang, F. Prevalence of venous thromboembolism in patients with severe novel coronavirus pneumonia. Journal of thrombosis and haemostasis : JTH 18, 1421-1424, doi:10.1111/jth.14830 (2020).

2 Lodigiani, C. et al. Venous and arterial thromboembolic complications in COVID-19 patients admitted to an academic hospital in Milan, Italy. Thrombosis research 191, 9-14, doi:10.1016/j.thromres.2020.04.024 (2020).

3 Lax, S. F. et al. Pulmonary Arterial Thrombosis in COVID-19 With Fatal Outcome: Results From a Prospective, Single-Center, Clinicopathologic Case Series. Annals of internal medicine, doi:10.7326/m202566 (2020).

4 Fox, S. E. et al. Pulmonary and cardiac pathology in African American patients with COVID-19: an autopsy series from New Orleans. The Lancet. Respiratory medicine, doi:10.1016/s2213-2600(20)302435 (2020).

5 Smeeth, L. et al. Risk of myocardial infarction and stroke after acute infection or vaccination. The New England journal of medicine 351, 2611-2618, doi:10.1056/NEJMoa041747 (2004).

6 Clayton, T. C., Thompson, M. \& Meade, T. W. Recent respiratory infection and risk of cardiovascular disease: case-control study through a general practice database. European heart journal 29, 96-103, doi:10.1093/eurheartj/ehm516 (2008).

7 Clayton, T. C., Gaskin, M. \& Meade, T. W. Recent respiratory infection and risk of venous thromboembolism: case-control study through a general practice database. International journal of epidemiology 40, 819-827, doi:10.1093/ije/dyr012 (2011).

8 Connors, J. M. \& Levy, J. H. COVID-19 and its implications for thrombosis and anticoagulation. Blood 135, 2033-2040, doi:10.1182/blood.2020006000 (2020).

9 Spyropoulos, A. C. et al. Scientific and Standardization Committee Communication: Clinical Guidance on the Diagnosis, Prevention and Treatment of Venous Thromboembolism in Hospitalized Patients with COVID-19. Journal of Thrombosis and Haemostasis n/a, doi:10.1111/jth.14929 (2020).

10 Moores, L. K. et al. Prevention, Diagnosis, and Treatment of VTE in Patients With Coronavirus Disease 2019: CHEST Guideline and Expert Panel Report. CHEST, doi:10.1016/j.chest.2020.05.559.

11 Liao, S. et al. Incidence of venous thromboembolism in different ethnic groups: a regional direct comparison study. Journal of Thrombosis and Haemostasis 12, 214-219, doi:10.1111/jth.12464 (2014). 
12 Zakai, N. A. et al. Racial and regional differences in venous thromboembolism in the United States in 3 cohorts. Circulation 129, 1502-1509, doi:10.1161/CIRCULATIONAHA.113.006472 (2014).

13 Lederle, F. A., Zylla, D., MacDonald, R. \& Wilt, T. J. Venous thromboembolism prophylaxis in hospitalized medical patients and those with stroke: a background review for an American College of Physicians Clinical Practice Guideline. Annals of internal medicine 155, 602-615, doi:10.7326/0003-4819155-9-201111010-00008 (2011).

14 Mortus, J. R. et al. Thromboelastographic Results and Hypercoagulability Syndrome in Patients With Coronavirus Disease 2019 Who Are Critically III. JAMA Network Open 3, e2011192-e2011192, doi:10.1001/jamanetworkopen.2020.11192 (2020).

15 Tan, C. W. et al. Critically ill COVID-19 infected patients exhibit increased clot waveform analysis parameters consistent with hypercoagulability. American journal of hematology 95, E156-E158, doi:10.1002/ajh.25822 (2020).

16 Wee, L. E. et al. Containing COVID-19 in the Emergency Department: The Role of Improved Case Detection and Segregation of Suspect Cases. Academic emergency medicine : official journal of the Society for Academic Emergency Medicine 27, 379-387, doi:10.1111/acem.13984 (2020).

17 Taylor, F. B., Jr., Toh, C. H., Hoots, W. K., Wada, H. \& Levi, M. Towards definition, clinical and laboratory criteria, and a scoring system for disseminated intravascular coagulation. Thrombosis and haemostasis 86, 1327-1330 (2001).

18 Iba, T., Nisio, M. D., Levy, J. H., Kitamura, N. \& Thachil, J. New criteria for sepsis-induced coagulopathy (SIC) following the revised sepsis definition: a retrospective analysis of a nationwide survey. BMJ Open 7, e017046-e017046, doi:10.1136/bmjopen-2017-017046 (2017).

19 Kwong, J. C. et al. Acute Myocardial Infarction after Laboratory-Confirmed Influenza Infection. New England Journal of Medicine 378, 345-353, doi:10.1056/NEJMoa1702090 (2018).

20 Bunce, P. E. et al. Pandemic H1N1 influenza infection and vascular thrombosis. Clinical infectious diseases : an official publication of the Infectious Diseases Society of America 52, e14-17, doi:10.1093/cid/ciq125 (2011).

21 Bilaloglu, S. et al. Thrombosis in Hospitalized Patients With COVID-19 in a New York City Health System. Jama, doi:10.1001/jama.2020.13372 (2020).

22 Middeldorp, S. et al. Incidence of venous thromboembolism in hospitalized patients with COVID19. Journal of thrombosis and haemostasis : JTH, doi:10.1111/jth.14888 (2020).

23 Jin-fu Xu, L. W., Lan Zhao et al. Risk assessment of venous thromboembolism and bleeding in COVID-19 patients. doi:https://doi.org/10.21203/rs.3.rs-18340/v1 (2020). 
24 Llitjos, J. F. et al. High incidence of venous thromboembolic events in anticoagulated severe COVID-19 patients. Journal of thrombosis and haemostasis : JTH 18, 1743-1746, doi:10.1111/jth.14869 (2020).

25 Klok, F. A. et al. Incidence of thrombotic complications in critically ill ICU patients with COVID-19. Thrombosis research 191, 145-147, doi:10.1016/j.thromres.2020.04.013 (2020).

26 Molina, J. A., Jiang, Z. G., Heng, B. H. \& Ong, B. K. Venous thromboembolism at the National Healthcare Group, Singapore. Annals of the Academy of Medicine, Singapore 38, 470-478 (2009).

27 Nicole Tran, H. \& Klatsky, A. L. Lower risk of venous thromboembolism in multiple Asian ethnic groups. Preventive medicine reports 13, 268-269, doi:10.1016/j.pmedr.2019.01.006 (2019).

28 Wang, K. L. et al. The diagnosis and treatment of venous thromboembolism in asian patients. Thrombosis journal 16, 4, doi:10.1186/s12959-017-0155-z (2018).

29 White, R. H. \& Keenan, C. R. Effects of race and ethnicity on the incidence of venous thromboembolism. Thrombosis research 123 Suppl 4, S11-17, doi:10.1016/s0049-3848(09)70136-7 (2009).

30 Vejpongsa, P. et al. Outcomes of Acute Myocardial Infarction in Patients with Influenza and Other Viral Respiratory Infections. Am J Med 132, 1173-1181, doi:10.1016/j.amjmed.2019.05.002 (2019).

31 Shi, S. et al. Association of Cardiac Injury With Mortality in Hospitalized Patients With COVID-19 in Wuhan, China. JAMA cardiology, doi:10.1001/jamacardio.2020.0950 (2020).

32 Tan, C. W. et al. Assessment of aPTT-based clot waveform analysis for the detection of haemostatic changes in different types of infections. Scientific Reports 10, 14186, doi:10.1038/s41598020-71063-1 (2020).

33 Ruberto, M. F. et al. The venous thromboembolic risk and the clot wave analysis: a useful relationship? Clinical chemistry and laboratory medicine 56, 448-453, doi:10.1515/cclm-2017-0678 (2018).

34 Goshua, G. et al. Endotheliopathy in COVID-19-associated coagulopathy: evidence from a singlecentre, cross-sectional study. The Lancet Haematology, doi:10.1016/S2352-3026(20)30216-7.

35 Connell, N. T., Battinelli, E. M. \& Connors, J. M. Coagulopathy of COVID-19 and antiphospholipid antibodies. Journal of thrombosis and haemostasis : JTH, 10.1111/jth.14893, doi:10.1111/jth.14893 (2020).

\section{Tables}


Table 1: Clinical characteristics, thrombotic events and outcomes of patients with SARS-CoV-2 and non-CoV-2 respiratory viruses.

$$
\begin{array}{lll}
\begin{array}{l}
\text { SARS-CoV-2 } \\
(n=182)
\end{array} & \begin{array}{l}
\text { Non-CoV-2 respiratory viruses } \\
(n=165)
\end{array} & \begin{array}{l}
P \text { - } \\
\text { values }
\end{array}
\end{array}
$$

\begin{tabular}{|c|c|c|c|}
\hline Age, Median (IQR) & $37(30,51)$ & $35(29,51)$ & 0.122 \\
\hline Gender - no.(\%) & & & $<0.001$ \\
\hline Male & $133(73.1)$ & $87(52.7)$ & \\
\hline Female & $49(26.9)$ & $78(47.3)$ & \\
\hline Race - no.(\%) & & & $<0.001$ \\
\hline Chinese & $63(34.6)$ & $74(44.8)$ & \\
\hline Malay & $6(3.3)$ & $31(18.8)$ & \\
\hline Indian & $81(44.5)$ & $35(21.2)$ & \\
\hline Others & $32(17.6)$ & $25(15.2)$ & \\
\hline \multicolumn{4}{|l|}{ Comorbidities - no.(\%) } \\
\hline Hypertension & $44(24.2)$ & $28(17.0)$ & 0.098 \\
\hline Hyperlipidemia & $15(8.20)$ & $24(18.50)$ & 0.063 \\
\hline Diabetes Mellitus & $10(5.5)$ & $22(13.3)$ & 0.012 \\
\hline Ischemic Heart Disease & $6(3.30)$ & $13(7.90)$ & 0.061 \\
\hline Prior stroke & $4(2.20)$ & $5(3.0)$ & 0.626 \\
\hline Renal disease & $1(0.50)$ & $10(6.10)$ & 0.003 \\
\hline Chronic lung disease & $18(9.90)$ & $15(9.10)$ & 0.800 \\
\hline Liver disease & $0(0.00)$ & $3(1.80)$ & 0.106 \\
\hline Active malignancy & $0(0.00)$ & $3(1.80)$ & 0.106 \\
\hline \multicolumn{4}{|c|}{ Charlson Comorbidity index } \\
\hline Median (IQR) & $0(0,1)$ & $0(0,1)$ & 0.39 \\
\hline $0-2$ points & $167(91.8)$ & $145(87.9)$ & 0.231 \\
\hline$\geq 3$ points & $15(8.2)$ & $20(12.1)$ & \\
\hline
\end{tabular}

\section{Demographics}

\section{Existing antithrombotic agents -} no.(\%)

Antiplatelet

8 (4.4)

$7(4.2)$

1.0 


\begin{tabular}{|llll|}
\hline Anticoagulation & $1(0.5)$ & $1(0.6)$ & 1.0 \\
\hline Thrombotic outcomes & & & 1.0 \\
\hline Arterial thrombosis - no.(\%) & $2(1.1)$ & $1(0.6)$ & - \\
\hline Venous thrombosis - no.(\%) & 0 & 0 & 0.6308 \\
\hline Event/1000 patient days & 1.0 & 1.8 & 0.035 \\
\hline Outcomes & & & 0.500 \\
\hline $\begin{array}{l}\text { Required O2 supplementation - } \\
\text { no.(\%) }\end{array}$ & $20(11.0)$ & $8(4.8)$ & 0.047 \\
\hline HD - no.(\%) & $2(1.1)$ & $0(0)$ & 0.073 \\
\hline ICU - no.(\%) & $9(4.9)$ & $2(1.2)$ & - \\
\hline LOS in ICU, median (IQR) & $20(9,27)$ & $5(3,7)$ & $<0.001$ \\
\hline Total patient days in ICU & 160 & 10 & - \\
\hline LOS, Median (IQR) & $7.5(6,13)$ & $3(2,3)$ & 1.0 \\
\hline Total patient days in hospital & 1979 & 554 & $1(0.6)$ \\
\hline Death - no.(\%) & $2(1.1)$ & & \\
\hline
\end{tabular}

Table 2: Paired comparison analysis of coagulation data (PT, aPTT and aPTT-based clot waveform analysis profiles) of patients with mild COVID-19^. These are intra-individual comparison of their serial coagulation data. 


\begin{tabular}{|c|c|c|c|c|c|}
\hline \multirow[t]{2}{*}{ Coagulation Tests } & \multicolumn{2}{|c|}{$1^{\text {st }}$ Time Point $(n=10)$} & \multicolumn{2}{|c|}{$2^{\text {nd }}$ Time Point $(n=10)$} & \multirow{2}{*}{$\begin{array}{l}\mathrm{P} \text { - } \\
\text { value }\end{array}$} \\
\hline & $\begin{array}{l}\text { Median } \\
\text { (IQR) }\end{array}$ & Range & $\begin{array}{l}\text { Median } \\
\text { (IQR) }\end{array}$ & Range & \\
\hline APTT, s & $31.98(3.65)$ & $\begin{array}{l}28.75- \\
36.05\end{array}$ & $30.70(3.70)$ & $\begin{array}{l}25.80- \\
36.90\end{array}$ & 0.066 \\
\hline Min1, \%/s & $5.04(1.76)$ & $3.67-7.04$ & $5.16(3.53)$ & $3.15-8.67$ & 0.386 \\
\hline $\operatorname{Min} 2, \% / s^{2}$ & $0.71(0.24)$ & $0.57-1.05$ & $0.77(0.62)$ & $0.47-1.35$ & 0.286 \\
\hline $\operatorname{Max} 2, \% / s^{2}$ & $0.56(0.18)$ & $0.44-0.85$ & $0.59(0.53)$ & $0.35-1.11$ & 0.259 \\
\hline PT, s & $10.20(0.70)$ & $9.70-10.90$ & $10.30(0.80)$ & $9.50-11.40$ & 0.777 \\
\hline \multirow{2}{*}{$\begin{array}{l}\text { Days since symptoms } \\
\text { onset }\end{array}$} & $7(5)$ & $5-17$ & $10.5(8)$ & $7-19$ & 0.005 \\
\hline & \multicolumn{2}{|c|}{$1^{\text {st }}$ Time Point $(n=7)$} & \multicolumn{2}{|c|}{$3^{\text {nd }}$ Time Point $(n=7)$} & \\
\hline APTT, s & $32.25(2.35)$ & $\begin{array}{l}28.75- \\
34.85\end{array}$ & $30.40(3.25)$ & $\begin{array}{l}28.00- \\
32.45\end{array}$ & 0.051 \\
\hline Min1, \%/s & $4.80(0.76)$ & $4.13-7.04$ & $4.25(1.04)$ & $2.85-8.17$ & 0.236 \\
\hline $\operatorname{Min} 2, \% / s^{2}$ & $0.70(0.14)$ & $0.61-1.05$ & $0.58(0.21)$ & $0.45-1.29$ & 0.310 \\
\hline $\operatorname{Max} 2, \% / s^{2}$ & $0.54(0.12)$ & $0.47-0.80$ & $0.44(0.24)$ & $0.35-1.05$ & 0.351 \\
\hline PT, s & $10.30(0.40)$ & $9.70-10.80$ & $10.80(0.80)$ & $\begin{array}{l}10.00- \\
11.40\end{array}$ & 0.058 \\
\hline \multirow[t]{2}{*}{$\begin{array}{l}\text { Days since symptoms } \\
\text { onset }\end{array}$} & $7(9)$ & $6-17$ & $14(8)$ & $11-22$ & 0.018 \\
\hline & \multicolumn{2}{|c|}{$1^{\text {st }}$ Time Point $(n=4)$} & \multicolumn{2}{|c|}{ Convalescence $(n=4)$} & \\
\hline APTT, s & $31.05(2.99)$ & $\begin{array}{l}30.05- \\
34.00\end{array}$ & $27.80(2.40)$ & $\begin{array}{l}27.40- \\
30.50\end{array}$ & 0.068 \\
\hline Min1, \%/s & $5.79(1.76)$ & $5.07-7.04$ & $5.90(2.62)$ & $4.76-7.53$ & 0.715 \\
\hline $\operatorname{Min} 2, \% / s^{2}$ & $0.90(0.261)$ & $0.78-1.05$ & $0.93(0.43)$ & $0.76-1.22$ & 0.465 \\
\hline $\operatorname{Max} 2, \% / s^{2}$ & $0.71(0.259)$ & $0.57-0.85$ & $0.76(0.39)$ & $0.61-1.04$ & 0.068 \\
\hline PT, s & $10.40(0.70)$ & $9.80-10.70$ & $10.25(1.20)$ & $9.70-11.30$ & 1.000 \\
\hline $\begin{array}{l}\text { Days since symptoms } \\
\text { onset }\end{array}$ & $8.5(9)$ & $4-15$ & $48(67)$ & $22-99$ & 0.068 \\
\hline
\end{tabular}


* No D-dimer and fibrinogen data presented as not more than 2 pairs of these results were available.

${ }^{\wedge}$ Mild COVID-19 defined as patients who did not require supplementary oxygen support throughout the course of infection.

Table 3: Inter-individual comparison of coagulation data (PT, aPTT and aPTT-based clot waveform analysis profiles) between patients with mild COVID-19^ and severe COVID-19\#.

\begin{tabular}{|c|c|c|c|c|c|}
\hline \multirow[t]{2}{*}{ Coagulation Tests } & \multicolumn{2}{|c|}{$\begin{array}{l}\text { Mild COVID-19 Disease } \\
(n=28)\end{array}$} & \multicolumn{2}{|c|}{$\begin{array}{l}\text { Severe COVID-19 Disease } \\
(n=14)\end{array}$} & \multirow[t]{2}{*}{$\begin{array}{l}\mathrm{P}- \\
\text { value }\end{array}$} \\
\hline & $\begin{array}{l}\text { Median } \\
\text { (IQR) }\end{array}$ & Range & $\begin{array}{l}\text { Median } \\
\text { (IQR) }\end{array}$ & Range & \\
\hline APTT, s & $\begin{array}{l}31.78 \\
(3.04)\end{array}$ & $\begin{array}{l}28.70- \\
40.20\end{array}$ & $36.85(7.38)$ & $\begin{array}{l}30.80- \\
42.10\end{array}$ & $<0.001$ \\
\hline Min1, \%/s & $5.05(1.38)$ & $3.63-8.54$ & $6.48(1.23)$ & $5.31-8.63$ & $<0.001$ \\
\hline $\operatorname{Min} 2, \% / s^{2}$ & $0.74(0.18)$ & $0.53-1.32$ & $0.92(0.29)$ & $0.67-1.31$ & 0.033 \\
\hline $\operatorname{Max} 2, \% / s^{2}$ & $0.57(0.13)$ & $0.39-1.04$ & $0.67(0.22)$ & $0.47-1.01$ & 0.085 \\
\hline PT, s & $\begin{array}{l}10.33 \\
(0.70)\end{array}$ & $9.70-13.20$ & $10.85(1.28)$ & $9.50-13.00$ & 0.180 \\
\hline D-dimer*, mg/L FEU & $0.43(0.64)$ & $0.19-5.15$ & $0.845(0.61)$ & $0.62-1.33$ & 0.104 \\
\hline Fibrinogen^, g/L & $3.08(1.01)$ & $2.23-3.44$ & $4.56(-)$ & $4.12-6.81$ & 0.057 \\
\hline $\begin{array}{l}\text { Days since symptoms } \\
\text { onset }\end{array}$ & $7(5)$ & $4-34$ & $9(8)$ & $4-24$ & 0.218 \\
\hline
\end{tabular}

* D-dimer results were only available in 11 and 4 patients with mild and severe COVID-19, respectively.

** Fibrinogen results were only available in 4 and 3 patients with mild and severe COVID-19, respectively.

${ }^{\wedge}$ Mild COVID-19 defined as patients who did not require supplementary oxygen support throughout the course of infection. 
\# Severe COVID-19 defined as patients who required supplementary oxygen support during course of infection

Table 4: Inter-individual comparison of coagulation data (PT, aPTT and aPTT-based clot waveform analysis profiles) between mild COVID-19 and non-CoV-2 respiratory viral infections ${ }^{\wedge}$, as well as between critically ill COVID-19 and non-CoV-2 respiratory viral infections". 


\begin{tabular}{|c|c|c|c|c|c|}
\hline \multirow[t]{2}{*}{ Coagulation Tests } & \multicolumn{2}{|c|}{ Mild COVID-19 $(n=28)$} & \multicolumn{2}{|c|}{$\begin{array}{l}\text { Mild Non -CoV-2 respiratory viruses } \\
(n=3)\end{array}$} & \multirow[t]{2}{*}{$\begin{array}{l}P \text { - } \\
\text { value }\end{array}$} \\
\hline & $\begin{array}{l}\text { Median } \\
\text { (IQR) }\end{array}$ & Range & Median (IQR) & Range & \\
\hline APTT, s & $\begin{array}{l}31.78 \\
(3.04)\end{array}$ & $\begin{array}{l}28.70- \\
40.20\end{array}$ & $29.60(-)$ & $25.80-33.30$ & 0.229 \\
\hline Min1, \%/s & $\begin{array}{l}5.05 \\
(1.38)\end{array}$ & $\begin{array}{l}3.63- \\
8.54\end{array}$ & $5.76(-)$ & $3.60-7.79$ & 0.729 \\
\hline $\operatorname{Min} 2, \% / s^{2}$ & $\begin{array}{l}0.74 \\
(0.18)\end{array}$ & $\begin{array}{l}0.53- \\
1.32\end{array}$ & $0.95(-)$ & $0.58-1.17$ & 0.503 \\
\hline $\operatorname{Max} 2, \% / s^{2}$ & $\begin{array}{l}0.57 \\
(0.13)\end{array}$ & $\begin{array}{l}0.39- \\
1.04\end{array}$ & $0.80(-)$ & $0.47-0.95$ & 0.385 \\
\hline PT, s & $\begin{array}{l}10.33 \\
(0.70)\end{array}$ & $\begin{array}{l}9.70- \\
13.20\end{array}$ & $10.20(-)$ & $9.80-10.60$ & 0.503 \\
\hline \multirow{2}{*}{$\begin{array}{l}\text { Days since symptoms } \\
\text { onset }\end{array}$} & $7(5)$ & $4-34$ & $7(-)$ & $3-18$ & 0.925 \\
\hline & \multicolumn{2}{|c|}{$\begin{array}{l}\text { Critically III COVID-19 } \\
(n=9)\end{array}$} & \multicolumn{2}{|c|}{$\begin{array}{l}\text { Critically III Non -CoV-2 respiratory } \\
\text { viruses }(n=2)\end{array}$} & \\
\hline APTT, s & $\begin{array}{l}40.20 \\
(6.40)\end{array}$ & $\begin{array}{l}33.30- \\
42.10\end{array}$ & $41.45(-)$ & $29.70-53.20$ & 1.000 \\
\hline Min1, \%/s & $\begin{array}{l}6.27 \\
(1.50)\end{array}$ & $\begin{array}{l}5.31- \\
8.63\end{array}$ & $4.57(-)$ & $3.17-5.97$ & 0.327 \\
\hline $\operatorname{Min} 2, \% / s^{2}$ & $\begin{array}{l}0.84 \\
(0.30)\end{array}$ & $\begin{array}{l}0.67- \\
1.31\end{array}$ & $0.48(-)$ & $0.23-0.73$ & 0.073 \\
\hline $\operatorname{Max} 2, \% / s^{2}$ & $\begin{array}{l}0.60 \\
(0.25)\end{array}$ & $\begin{array}{l}0.47- \\
1.01\end{array}$ & $0.39(-)$ & $0.21-0.57$ & 0.218 \\
\hline PT, s & $\begin{array}{l}10.90 \\
(1.20)\end{array}$ & $\begin{array}{l}9.50- \\
13.0\end{array}$ & $11.10(-)$ & $10.70-11.50$ & 0.727 \\
\hline Platelet, $\times 10^{9} / \mathrm{L}$ & $199(127)$ & $74-360$ & $271(-)$ & $137-404$ & 0.727 \\
\hline $\begin{array}{l}\text { Days since symptoms } \\
\text { onset }\end{array}$ & $10(11)$ & $4-24$ & $13(-)$ & $12-14$ & 0.582 \\
\hline
\end{tabular}

* No D-dimer and fibrinogen data presented as these results were not available in the mild non-CoV-2 respiratory viruses group.

${ }^{\wedge}$ Mild COVID-19 and non-CoV-2 respiratory viral infections defined as patients who did not require supplementary oxygen support throughout course of infection 
\# Critically ill COVID-19 and non-CoV-2 respiratory viral infections defined as patients who required intensive care unit support

\section{Figures}

a.

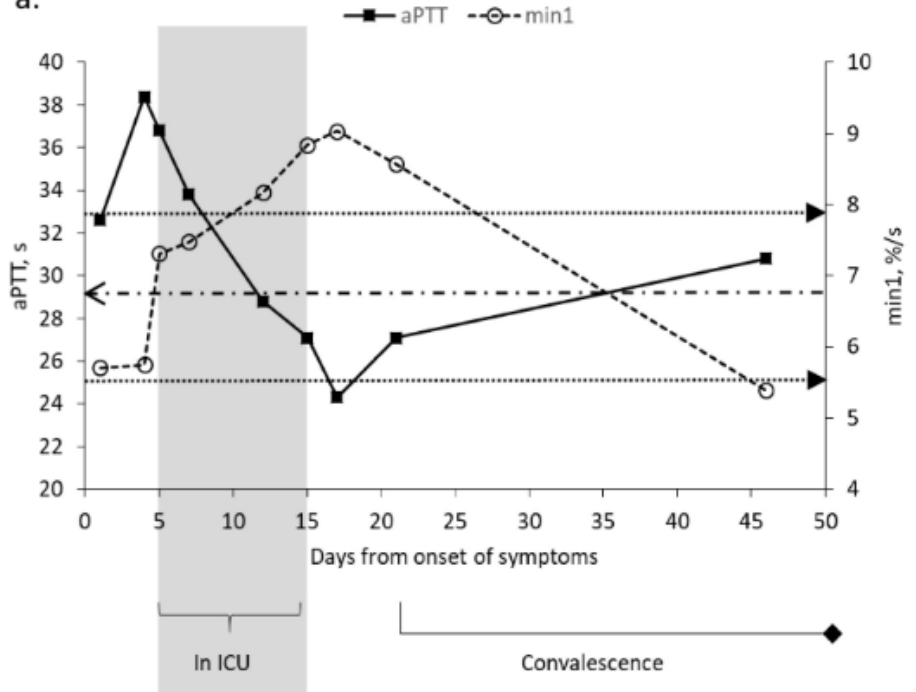

b.

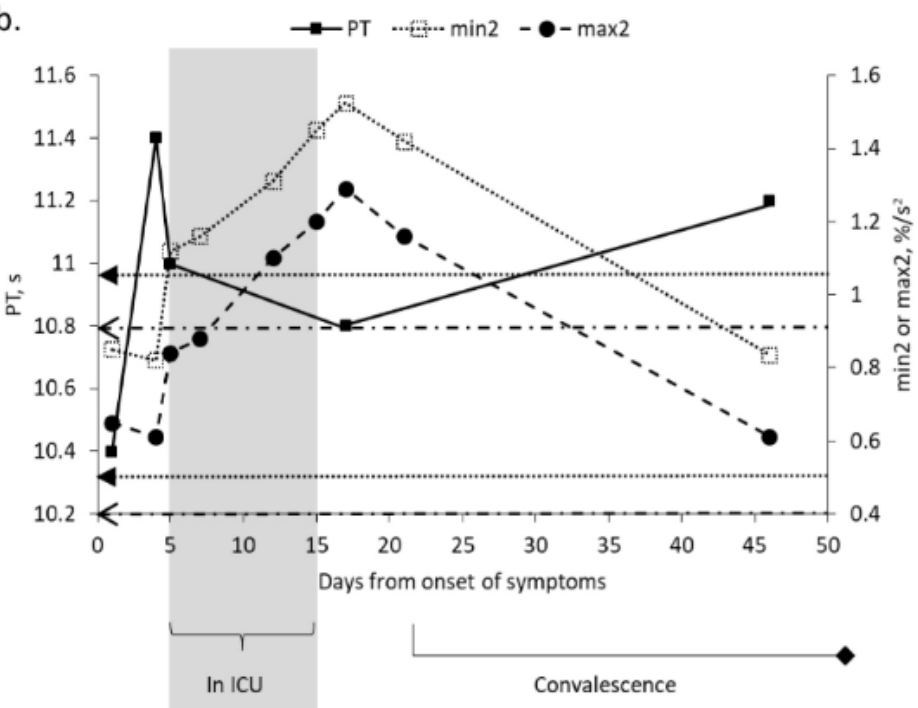

(a) Serial aPTT and min1 data of the patient. The horizontal dotted arrows $(\rightarrow$ depict the upper and lower limits of the reference intervals of aPTT while the horizontal dashed arrow $(<-)$ shows the upper limit of min1 reference interval ( $\min 1$ lower limit falls below the boundary of the graph shown). (b) Serial PT, min2 and max 2 data of the patient. All the PT results fall within the reference intervals of $9.9 \mathrm{~s}$ to $11.4 \mathrm{~s}$. The horizontal dashed arrows $(\ll-)$ depict the upper and lower limits of the reference intervals of min 2 while the horizontal dotted arrows $(\longleftarrow)$ show the upper and lower limits of max 2 reference intervals.

${ }^{*}$ Clinical deterioration defined as requiring intensive care unit (ICU) support

\section{Figure 1}

Dynamic changes of the serial haemostatic profiles of a COVID-19 patient from initial presentation to clinical deterioration* to subsequent recovery and convalescent phase.

\section{Supplementary Files}

This is a list of supplementary files associated with this preprint. Click to download.

- Supplementarytables.docx 